\title{
A Novel Sidelobe Cancellation Method for Binary Barker Code Pulse Compression
}

\author{
\{Abd El-Rahman H. Elbardawiny*, Ahmed Sobhy ${ }^{\dagger}$, FathyM. Ahmed, \\ and Mamdouh Hassan $\}^{\ddagger}$
}

\begin{abstract}
Barker Phase coded signals are one of the most effective technique used in pulse compression radars. The main problem of using these signals is the existence of sidelobes at the output of the matched filter. These sidelobes mask nearby weak targets. Also, it degrades the overall detection performance. In the present work, a novel method to totally remove these sidelobes is presented rather than conventional sidelobes reduction methods. The superior of the proposed method is evaluated through the Receiver Operation Characteristic (ROC) curve and simulated in case of single or multiple targets scenarios.
\end{abstract}

Keywords: Barker Code, Pulse Compression, Phase Coded Signals

\section{Introduction}

Pulse compression originally used to amplify the transmitted average power by temporal compression. It is a method that combines the energy of a long pulse width to obtain high resolution of a short pulse width in reception. By using pulse modulation method, each part of the transmitted pulse has unique frequency FM (frequency modulation) or phase PM (phase modulation). The received echo is processed in the receiver by the matched filter to readjusts the relative phases or frequency components so that a narrow or compressed pulse is again produced. The radar therefore obtains a better maximum range because of the increasing in average power and better range resolution because of the pulse compression resulted [1][2]. When a target echo signal is passed through a matched filter it outputs a spike-like main lobe and some unwanted sidelobes[3]. These sidelobes can form spurious targets or mask the main lobe of weak target echo signals at adjacent range cells [2]. To prevent these problems, enormous researches had been dedicated to reduce this unwanted sidelobes and obtain a maximum peak to sidelobe ratio (PSLR). In general, there are two main methods for sidelobe reduction. One is designing a mismatched filter directly from codes. The latest known solution of this method is the mismatched filter described by Adly T- Fam [4] [5]. The other way is employing an additional weighting filter after the matched filter. One possibility of this method is conventionally called the (R-G) filters [6]-[8].

In this paper we summarized the conventional algorithms of sidelobe reduction, followed by the new technique. The radar performance due to the proposed technique is compared with known published techniques.

\footnotetext{
Bardawiny@mtc.edu.com eng.ahmed3d@gmail.com Egyptian Armed Forces, Egypt.
} 


\section{Barker Code}

The binary code consists of a sequence of In-phase $\left(+1\left(0^{\circ}\right)\right)$ and out of phase $\left(-1\left(180^{\circ}\right)\right)$. Since the transmitted frequency is usually not a multiple of the reciprocal of the sub pulse width, the coded signal is generally discontinuous at the phase-reversal points. The binary choice of 0 or $\pi$ phase for each sub-pulse may be made at random. However, some random selections may be better suited than others for radar application. One criterion for the selection of a good phasecoded waveform is that its autocorrelation function should have equal time low side-lobe as shown in Fig. 1. In Table 1, the binary phase-coded sequence of $0, \pi$ values that result in equal minimum side-lobes after passes through the matched filter is called a Barker codes.

Table 1 Barker codes

\begin{tabular}{c|c}
\hline \hline Code Length & Code \\
\hline 2 &,+--+ \\
\hline 3 & ++- \\
\hline 4 &,+-+++--- \\
\hline 5 & +++-+ \\
\hline 7 & +++--+- \\
\hline 11 & +++--+--+- \\
\hline 13 & +++++--++-+-+ \\
\hline \hline
\end{tabular}

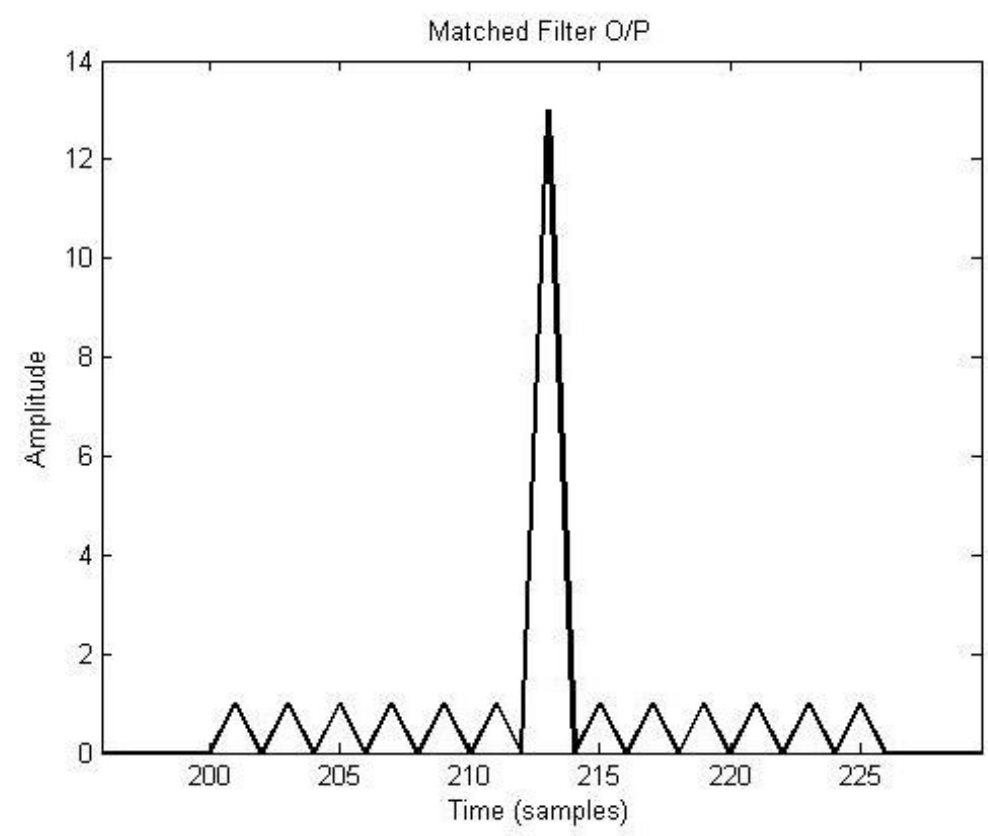

Fig. 1. Auto Correlation function (ACF) of 13 bit Barker code

\section{Sidelobe Reduction Techniques}

The mismatched filter [4][5] is designed by using the least mean squares criterion to design a sidelobe reduction mismatched filter. This filter follows the matched filter with multiple stages (Fig. 2.) to reduce the sidelobe level.

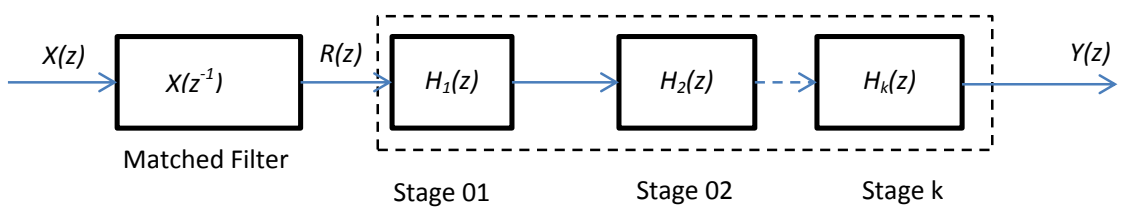

Fig. 2. Mismatched filter block diagram [4][5] 


\section{A. Mismatched Filter}

The Auto Correlation Function (ACF) of matched filter:

$$
\mathrm{R}(\mathrm{z})=\mathrm{X}(\mathrm{z}) \mathrm{X}\left(\mathrm{z}^{-1}\right)
$$

The waveform $R(Z)$ can be represented as a sum of a part representing the main lobe $(N)$ and a part representing the plurality of sidelobes $S(Z)$. So, $R(Z)$ is denoted by:

$$
\mathrm{R}(\mathrm{z})=\mathrm{N}+\mathrm{S}(\mathrm{z})
$$

The transfer function of the first stage of the filter is given by:

The output of the filter in (3) is:

$$
\mathrm{H}_{1}(\mathrm{z})=\mathrm{N}-\mathrm{S}(\mathrm{z})
$$

$$
\mathrm{Y}_{1}(\mathrm{z})=(\mathrm{N}+\mathrm{S}(\mathrm{z})) \times(\mathrm{N}-\mathrm{S}(\mathrm{z}))=\mathrm{N}^{2}-[\mathrm{S}(\mathrm{z})]^{2}
$$

The transfer function of the second stage of the filter is given by:

$$
\mathrm{H}_{2}(\mathrm{z})=\mathrm{N}^{2}+[\mathrm{S}(\mathrm{z})]^{2}
$$

If $\mathrm{k}$ stages are used, the transfer function of the kth stage is given by:

$$
\mathrm{H}_{\mathrm{k}}(\mathrm{z})=\mathrm{N}^{2^{(\mathrm{k}-1)}} \times[\mathrm{S}(\mathrm{z})]^{2^{(\mathrm{k}-1)}}
$$

\section{B. R-G Filter}

The (R-G filter) as shown in Fig. 3 was introduced by Rihaczek and Golden[6] to reduce Barker code sidelobes.

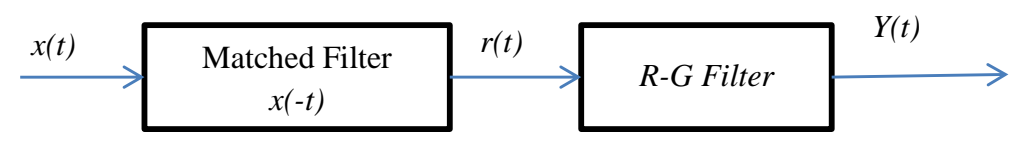

Fig. 3. General block diagram of R-G Filter [6]

The Autocorrelation function ACF $\mathrm{r}(\mathrm{t})$ could be considered as two sub functions (main lobe function $\mathrm{rm}(\mathrm{t})$ and sidelobers $(\mathrm{t})$ ). Each sub function has its Fourier transform. The convolution of the two functions are:

$$
r(t)=r_{m}(t) * r_{s}(t)
$$

The Fourier transform of equation (7)is:

where

$$
E(f)=E m(f) E s(f)
$$

$$
\begin{gathered}
E_{m}(f)=\frac{\sin ^{2}(\pi f T)}{(\pi f T)^{2}} \\
E_{s}(f)=N-1+\frac{\sin (2 \pi f N T)}{\sin (2 \pi f T)}
\end{gathered}
$$

The inverse of the $E_{s}(f)$ will be:

$$
H(f)=\frac{1}{E_{S}(f)}=\left[N-1+\frac{\sin (2 \pi f N T)}{\sin (2 \pi f T)}\right]^{-1}
$$

To analyze filter transfer function, $\mathrm{H}$ (f) can be approximated as a geometric series and selecting the first four terms of the $\mathrm{H}(\mathrm{f})$ as:

$$
H(f)=A+B \frac{\sin (2 \pi f N T)}{\sin (2 \pi f T)}+C\left[\frac{\sin (2 \pi f N T)}{\sin (2 \pi f T)}\right]^{2}+D\left[\frac{\sin (2 \pi f N T)}{\sin (2 \pi f T)}\right]^{3}
$$

where A,B,C,D are coefficients of the $(R-G)$ filter. So the filter transfer function with $\mathrm{m}$ coefficients is called as (R-G-m) stage filter. Trials for improving the (R-G filter) performance were done in [7]-[9]. 


\section{The Proposed Optimum Filter}

The proposed filter as shown in Fig. 4 is designed to follow the matched filter to completely remove sidelobes of ACF of barker coded signal.

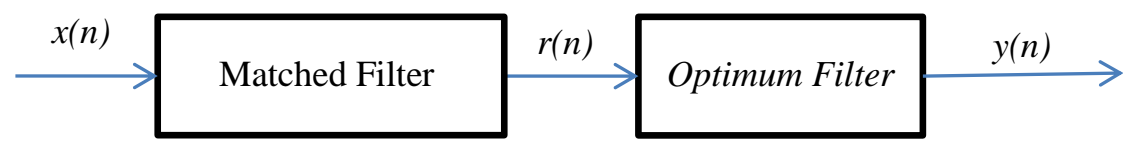

Fig. 4. General block diagram of proposed optimum filter

\section{Proposed Filter Transfer Function}

To investigate the analysis of the proposed filter, the ACF of the 7-elements Barker code is found to be:

$$
\mathrm{r}(\mathrm{n})=[-1,0,-1,0,-1,0,7,0,-1,0,-1,0,-1]
$$

The representation of (11) in frequency domain:

$$
\begin{gathered}
R\left(e^{j \omega}\right)=\left(-1-e^{-12 j \omega}\right)+0+\left(-e^{-2 j \omega}-e^{-10 j \omega}\right)+0+\left(-e^{-4 j \omega}-e^{-8 j \omega}\right)+0+ \\
7 e^{-6 j \omega}
\end{gathered}
$$

Simplifying equation (12) results:

$$
R\left(e^{j \omega}\right)=e^{-6 j \omega}[7-2 \cos 2 \omega-2 \cos 4 \omega-2 \cos 6 \omega]
$$

Equation (13) contains two parts, the main lobe, and the sidelobes, namely:

Where $\mathrm{M}\left(e^{j \omega}\right)$ represent main lobe

$$
\mathrm{R}\left(e^{j \omega}\right)=\mathrm{M}\left(e^{j \omega}\right)+\mathrm{S}\left(e^{j \omega}\right)
$$

$\mathrm{S}\left(e^{j \omega}\right)$ represent sidelobes

For the 7-elements Barker Code $\mathrm{M}\left(e^{j \omega}\right)$ is given by:

$$
\mathrm{M}\left(e^{j \omega}\right)=7 e^{-6 j \omega}
$$

However, the transfer function of proposed filter is:

Substitution for $\mathrm{M}$ and $\mathrm{R}$ yields to:

$$
H_{o p}\left(e^{j \omega}\right)=\mathrm{M}\left(e^{j \omega}\right) *\left[\mathrm{R}\left(e^{j \omega}\right)\right]^{-1}
$$

$$
H_{o p_{7}}\left(e^{j \omega}\right)=\frac{7}{7-2 \cos 2 \omega-2 \cos 4 \omega-2 \cos 6 \omega}
$$

The generalization of (17) for any Barker code length $(\mathrm{N})$ is:

where $\alpha_{\mathrm{i}}$ is the sidelobe coefficients

$$
H_{o p_{N}}\left(e^{j \omega}\right)=\frac{N}{N+2 \sum_{i=1}^{\frac{N-1}{2}} \alpha_{i} \cos 2 i \omega}
$$

\section{Simulation Results}

To investigate the performance of the proposed filter in the presence of normal Gaussian noise and noiseless cases, the 13-elements Barker coded signal applied to the system that is shown in Fig. 4. CFAR processor is used with probability of false alarm $\left(\mathrm{P}_{\mathrm{fa}}=10^{-6}\right)$ for target detection.

\section{A. Single Target}

When the noise free 13-elements Barker coded signal is used as the input to the system shown in Fig. 4, the output is sidelobe free as shown in Fig. 5. 


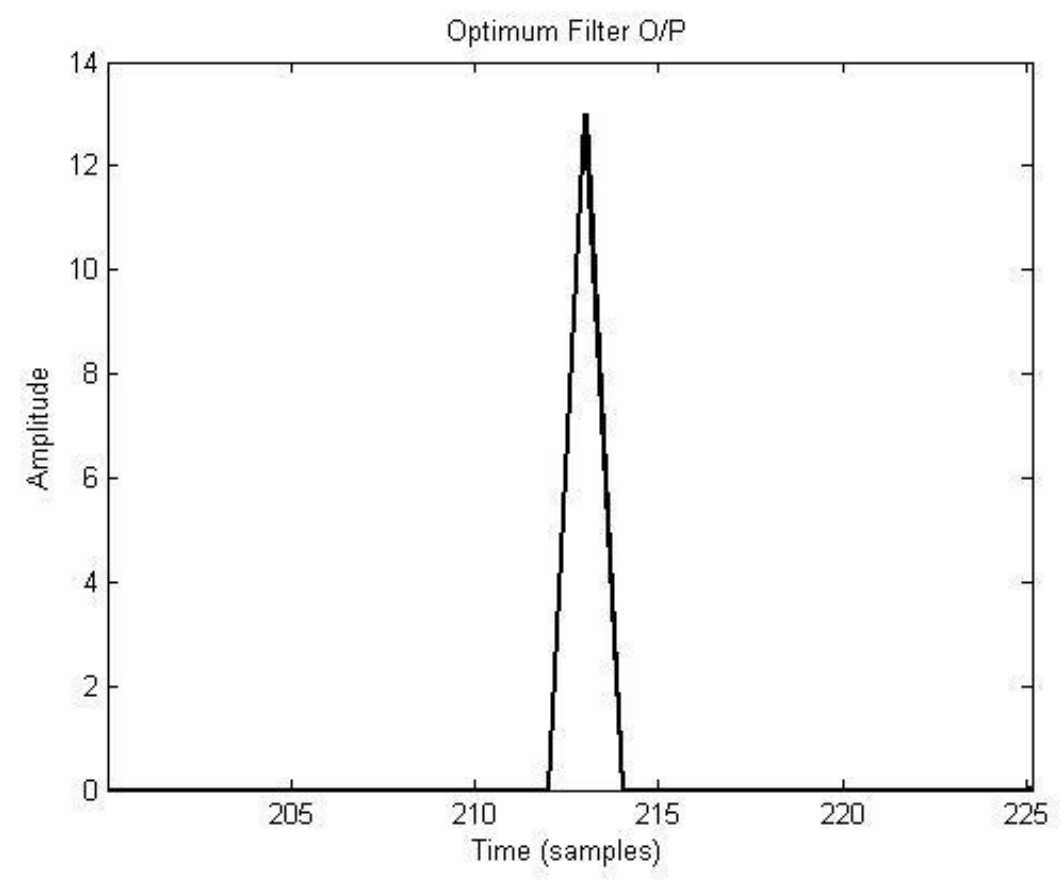

Fig. 5. Optimum filter output

Adding normal Gaussian noise with $(\mathrm{S} / \mathrm{N}=10 \mathrm{~dB})$ to the 13-elements Barker coded signal, the output from the matched filter is found to have a grass of high level as well as the CFAR processor threshold level is high as shown in Fig. 6.

Passing this output to the proposed optimum filter, it removes the high grass as shown in Fig. 7. The CFAR processor indicates the effect of the proposed optimum filter that it totally removes the sidelobes.

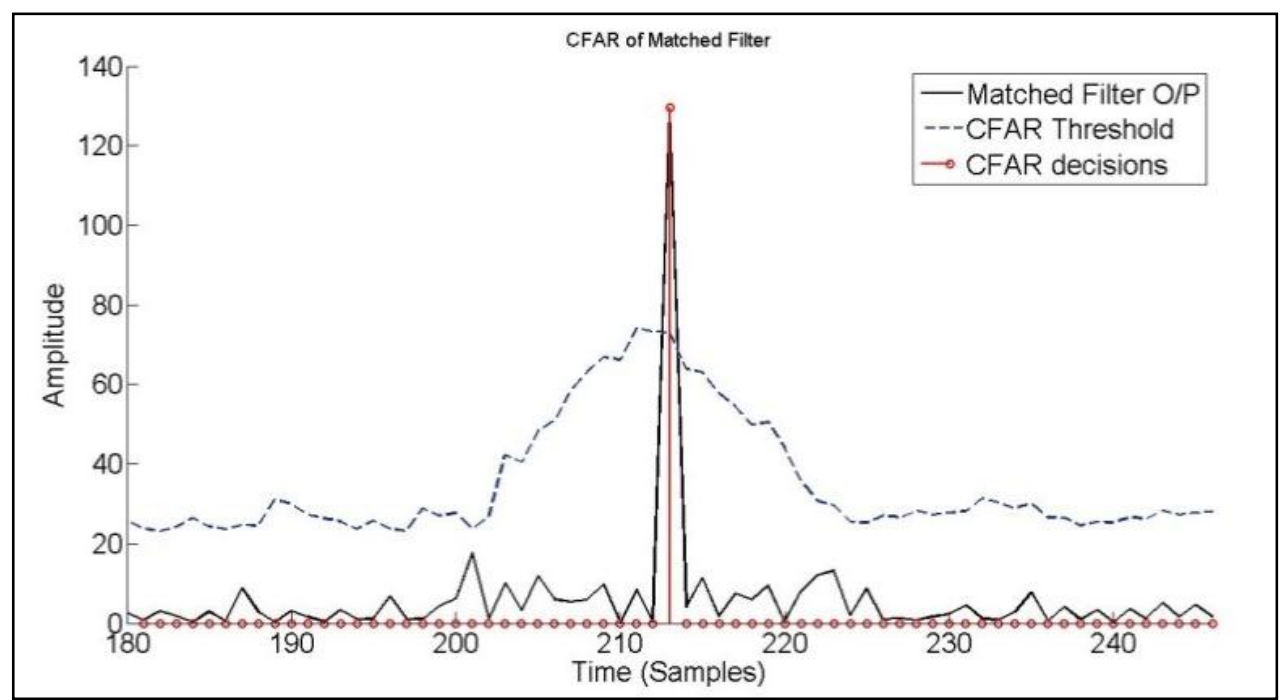

Fig. 6. CFAR detection after the matched filter $-\mathrm{S} / \mathrm{N}=10 \mathrm{~dB}-\mathrm{P}_{\mathrm{fa}}=10^{-06}$ 


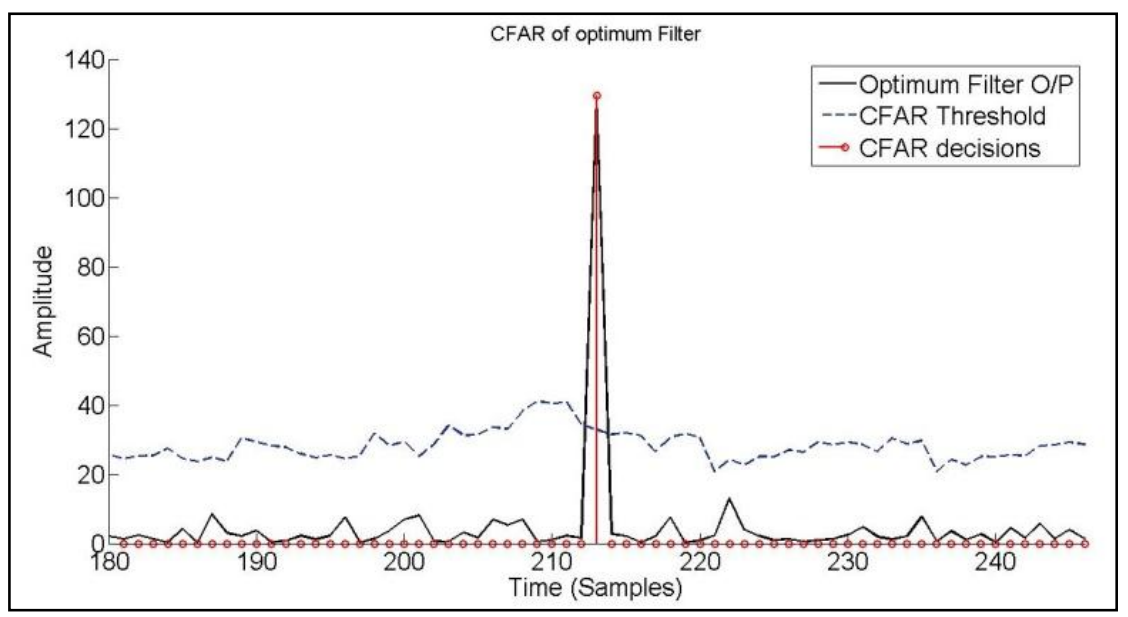

Fig. 7. CFAR detection after the Optimum Filter $-\mathrm{S} / \mathrm{N}=10 \mathrm{~dB}-\mathrm{P}_{\mathrm{fa}}=10^{-06}$

\section{B. Two Close Targets}

In the following section, the performance of the optimum filter in case of presence of two close targets is presented. The first target has $(\mathrm{S} / \mathrm{N}=10 \mathrm{~dB})$ while the second one has $(\mathrm{S} / \mathrm{N}=5 \mathrm{~dB})$. Fig. 8 shows that the target with smaller $\mathrm{S} / \mathrm{N}$ is miss detected when the matched filter is used alone.

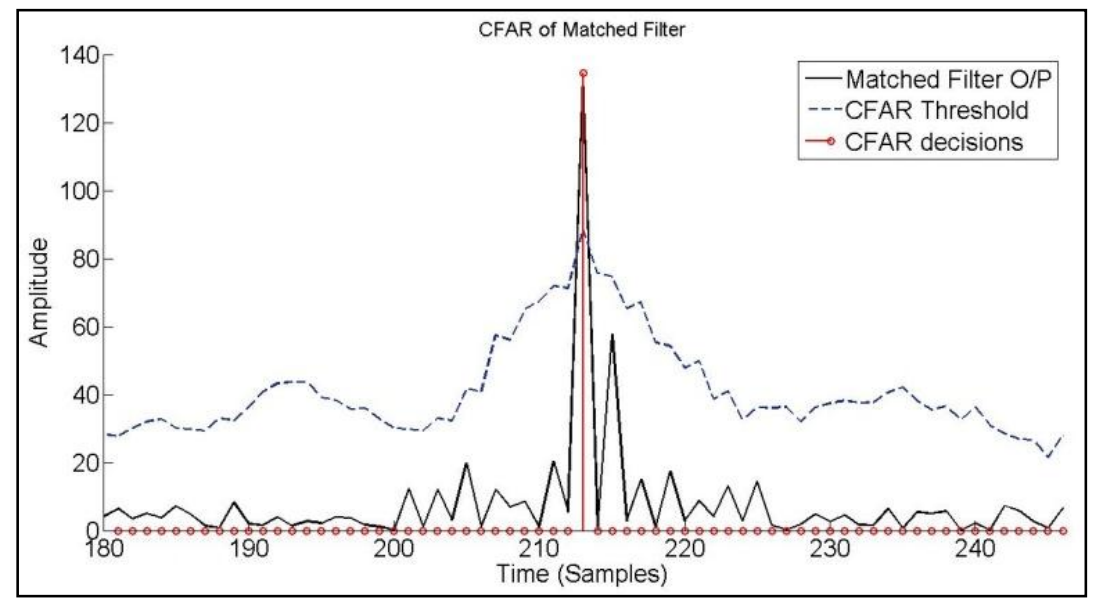

Fig. 8. CFAR detection $\left(\mathrm{P}_{\mathrm{fa}}=10^{-06}\right)$ of two close targets $(\mathrm{S} / \mathrm{N}=10 \mathrm{~dB}$ and $5 \mathrm{~dB})$ using matched filter alone

While Fig. 9 shows that the target with smaller $\mathrm{S} / \mathrm{N}$ is detected when the proposed optimum filter used. 


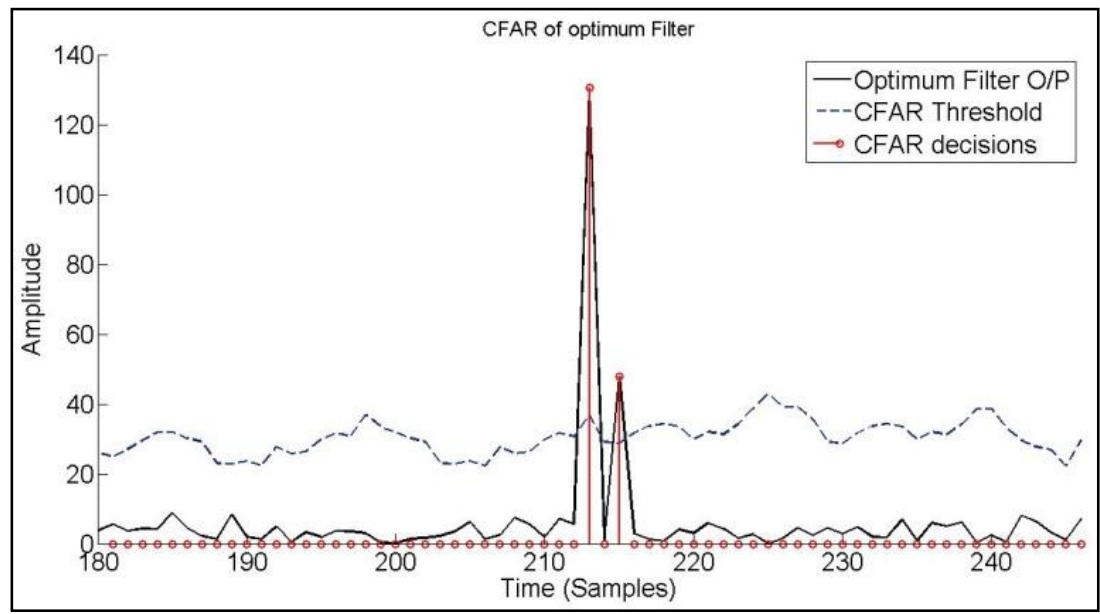

Fig. 9. CFAR detection $\left(\mathrm{P}_{\mathrm{fa}}=10^{-06}\right)$ of two close targets $(\mathrm{S} / \mathrm{N}=10 \mathrm{~dB}$ and $5 \mathrm{~dB})$ using matched filter together with optimum filter

Consider an extreme case in which the two close targets have high $\mathrm{S} / \mathrm{N}$ (15dB and 10dB respectively). Fig. 10 shows that when using the matched filter alone, the CFAR detects only one of the two targets together with one of the sidelobes. Meanwhile, Fig. 11 shows that the optimum filter rejected all the sidelobes and detected the two targets.

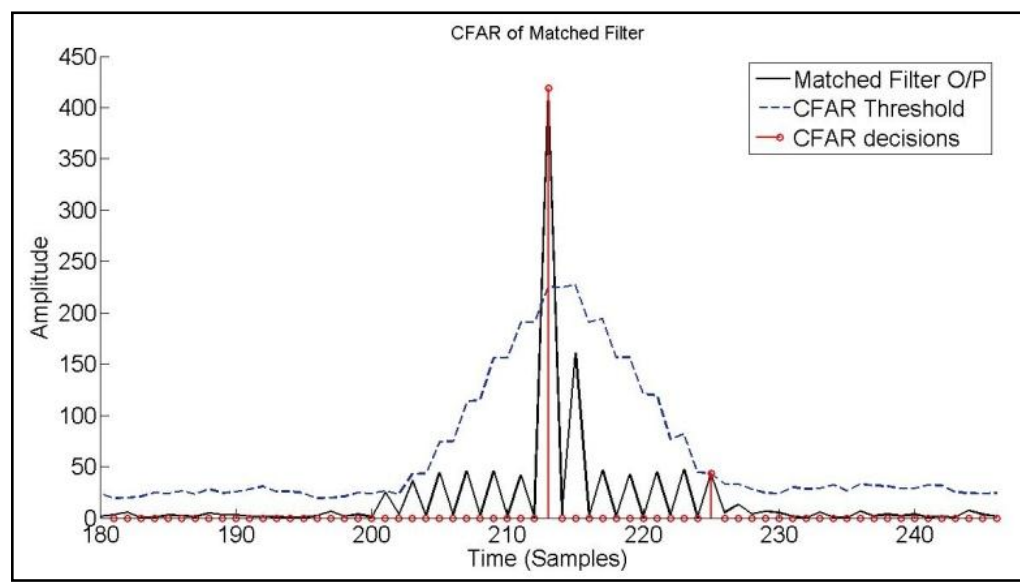

Fig. 10. CFAR detection $\left(\mathrm{P}_{\mathrm{fa}}=10^{-06}\right)$ of two close targets $(\mathrm{S} / \mathrm{N}=15 \mathrm{~dB}$ and $10 \mathrm{~dB})$ using matched filter.

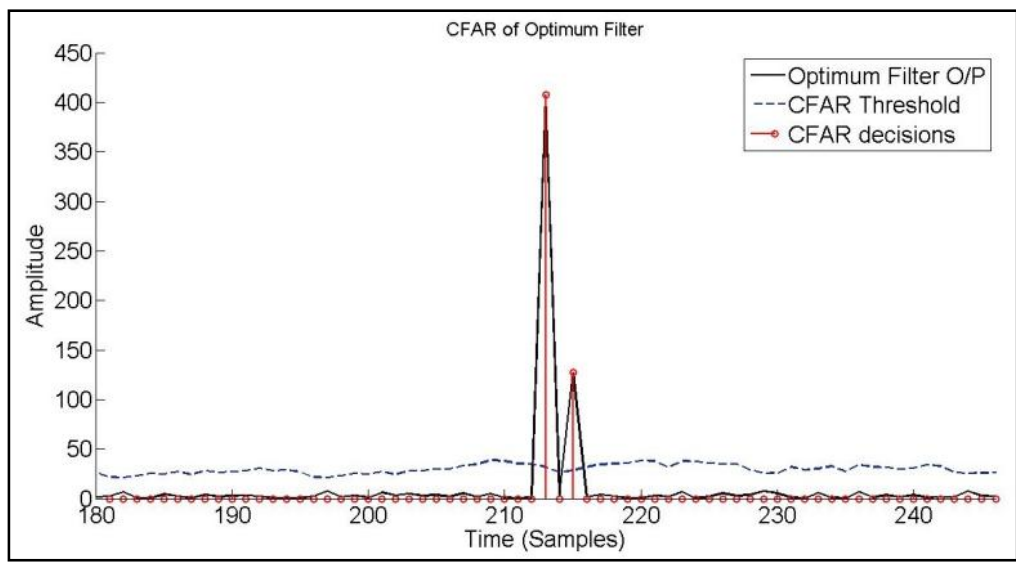

Fig. 11. CFAR detection $\left(\mathrm{P}_{\mathrm{fa}}=10^{-06}\right)$ of two close targets $(\mathrm{S} / \mathrm{N}=15 \mathrm{~dB}$ and $10 \mathrm{~dB})$ using matched filter together with optimum filter 


\section{ROC Curves}

The presence of the sidelobes will limit the (PSLR) in the output of the matched filter. Using matched filter only, as the S/N increases the sidelobes also increase with the same ratio. In our proposed optimum filter, the PSLR is almost linearly increases with the increase in S/N due to the absent of all sidelobes as shown in Fig. 12.

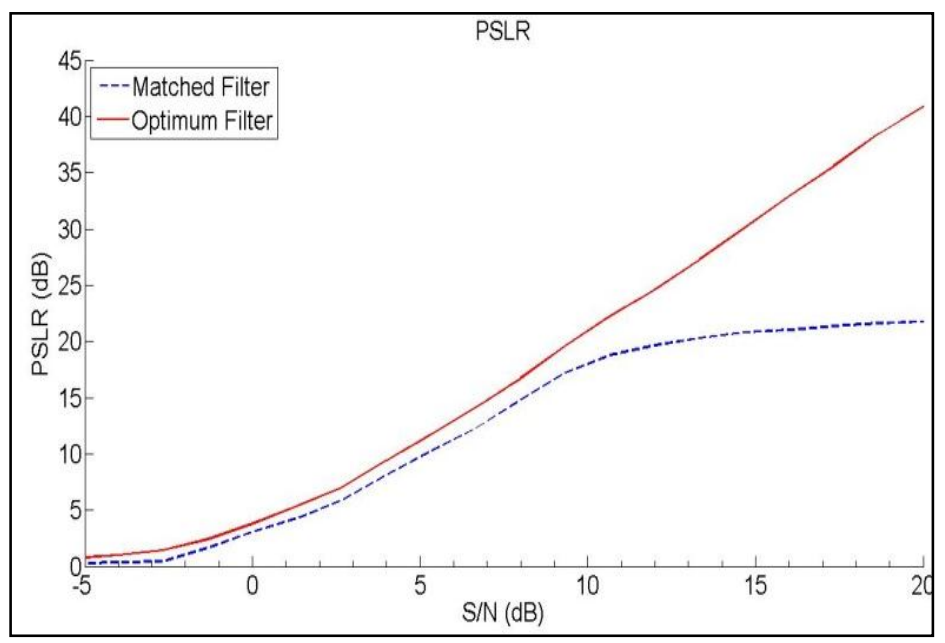

Fig. 12. Effect of increasing S/N on the PSLR in the output of matched filter and optimum filter

As a result of removing the sidelobes the probability of detection has increased. Fig. 13 shows the enhancement in probability of detection using the proposed optimum filter compared to the case of using matched filter alone.

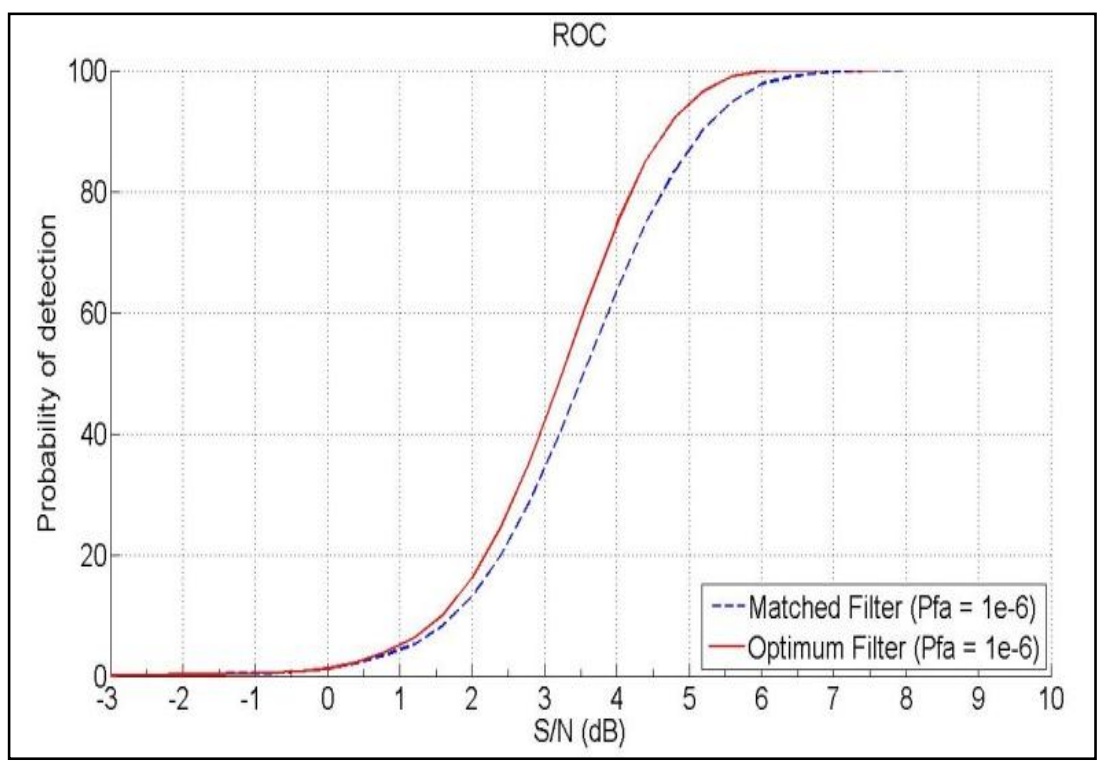

Fig. 13. ROC Curve 


\section{Evaluation of Proposed Filter}

Table 2 shows comparison between the known sidelobe reduction techniques [4][10][11] and the proposed filter for the 13-elements Barker code.

Table 2 PSLR for 13-Elements Barker Coded Signal from Conventional Algorithms

\begin{tabular}{c|c}
\hline Filter & PSLR (in dB) \\
\hline Matched Filter alone & 22.28 \\
Simple cascaded mismatched Filter [4] & 62.02 \\
Modified mismatched Filter -[4] & 321.85 \\
(R-G-2) [10] & 36.18 \\
(R-G-3) [10] & 38.3 \\
1 bit shift code [10] & 52.62 \\
2 bit shift code [10] & 44.14 \\
(R-G-2)LS[11] & 45.48 \\
(R-G-2)LP[11] & 46.35 \\
Factored Multiplicative Mismatched Filter[11] & 61.88 \\
(R-G-7)LP[11] & 111.80 \\
Proposed Optimum Filter & 320 \\
\hline
\end{tabular}

\section{Conclusion}

In the present work, a novel method for sidelobe cancellation at the output of matched filter of Barker coded signals is introduced. The proposed method achieved a PSLR of $320 \mathrm{~dB}$ which is considered as an optimum value compared to $111.8 \mathrm{~dB}$ for (R-G-7)LP [10] and much less in complexity of realization compared to the five stages of modified mismatched filter [4]. The Proposed technique has enhanced the detection of weak nearby targets in multiple target detection compared to standard matched filter. This system has been implemented on FPGA chip.

\section{References}

[1] Skolnik, M. I., "Introduction to Radar Systems (3rd ed.)." New York: McGraw-Hill, 2001, 339-369.

[2] Skolnik, M. I. "Radar Handbook (2nd ed.)”. McGraw-Hill, 1990, 10-15.

[3] Barker, R. H., "Group synchronization of binary digital systems," In W. Jackson (Ed.), Communication Theory, Burlington, MA: Academic Press, 1953.

[4] Adly T- Fam, "Mismatched Filter" United States Patent, Patent N0.: US 7,843,382 B2.

[5] Indranil Sarkar and Adly T. Fam, "Factored Multiplicative Mismatched Filters for Compound Barker Codes". Publications of the IEEE 1-4244-0284-0/07.

[6] Rihaczek, A. W. and Golden, R. M." Range sidelobe suppression for Barker codes.” IEEE Transactions on Aerospace and Electronic Systems, AES-7 (Nov. 1971), 1087-1092.

[7] Zoraster, S., "Minimum peak range sidelobe filters for binary phase coded waveforms. IEEE Transactions on Aerospace and Electronic Systems", AES-16 (Jan. 1980), $112-115$. 
[8] Hua, C. X. and Oksman, J., "A new algorithm to optimize Barker code sidelobe and suppression filter", IEEE Transactions on Aerospace and Electronic Systems, 26 (July 1990), 673-677.

[9] K.T. Jung, C.J. Kim, C.H. Lim, H.S. Lee, Y.K. Kwag, "Design of optimum mean square sidelobe suppression filters for Barker codes.”, In Proceedings of the IEEE International Radar Conference, Oct. 1992, 530-533.

[10] B. Kiranmai and P. Rajesh Kumar, "Performance Evaluation of Barker Codes using New Pulse Compression Technique", International Journal of Computer Applications (0975 8887) Volume 107 - No. 20, December 2014.

[11] Amirmokhtar Akbaripour and Mohammad H. Bastani, "Range Sidelobe Reduction Filter Design for Binary Coded Pulse Compression System", IEEE Transactions on Aerospace and Electronic Systems Vol. 48, No. 1 January 2012. 